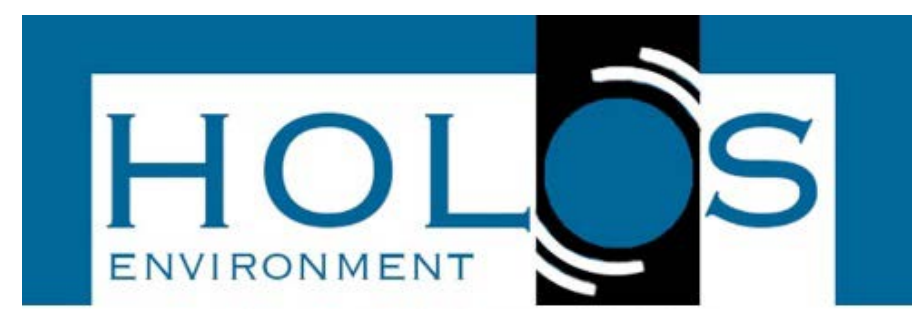

\title{
ABORDAGEM DO GERENCIAMENTO DE RESÍDUOS DE SERVIÇOS DE SAÚDE EM DROGARIAS E FARMÁCIAS DE MANIPULAÇÃO EM CIDADE DE PEQUENO PORTE DA REGIÃO SUL DO BRASIL
}

\author{
APPROACH OF HEALTH CARE WASTE MANAGEMENT IN DRUGSTORES AND \\ HANDLING PHARMACIES IN A SMALL TOWN OF THE SOUTHERN REGION OF \\ BRAZIL
}

\author{
Leila Dal Moro ${ }^{1}$; Adalberto Pandolfo ${ }^{1}$; Marcele Salles Martins ${ }^{1}$; Aline Pimentel Gomes ${ }^{1}$; \\ Laércio Stolfo Maculan²
}

Artigo recebido em: 06/06/2016 e aceito para publicação em: 16/10/2017.

DOI: http://dx.doi.org/10.14295/holos.v17i1.11478

Resumo: O presente trabalho aborda a problemática dos resíduos de serviços de saúde (RSS) gerados nas drogarias e farmácias de manipulação em uma cidade de pequeno porte do sul do Brasil, o gerenciamento desses resíduos visa à minimização dos impactos ambientais causados pelos materiais utilizados nos serviços da área da saúde descartados incorretamente no meio ambiente como, por exemplo, seringas e agulhas. Uma das principais causas do crescimento progressivo dos resíduos desses estabelecimentos é o uso contínuo e crescente de materiais descartáveis, que podem, com maior ou menor intensidade, causar a contaminação da qualidade da água e gerar poluição. O objetivo do estudo foi apresentar a situação do processo de gerenciamento dos RSS nas drogarias e farmácias de manipulação na cidade em estudo, relacionando à legislação vigente. As informações foram coletadas sob a forma de entrevistas e preenchimento de um questionário. Os resultados mostram em relação à quantidade de resíduos que a maioria das empresas geram aproximadamente 15 litros/mês, sendo compostos por: medicamentos vencidos, material injetável, frascos danificados, EPi's e resíduos de matéria prima. Foi observado o perfil das empresas, acerca dos procedimentos adotados quanto à disposição final, segregação, treinamentos oferecidos aos colaboradores, a quantificação dos acidentes envolvendo os resíduos e a presença de plano de gerenciamento de resíduos.

Palavras-chave: Geração de resíduos. Minimização de impactos ambientais. Processo de gerenciamento.

Abstract: This paper discusses the problem of healthcare waste generated in drugstores and handling pharmacies in a small town, aiming at the minimization of environmental impacts caused by the materials used in healthcare services incorrectly discarded in the environment as, for example, syringes and needles. One of the main causes of the progressive growth of the waste is the continued and increasing use of disposables, which can, with greater or lesser intensity, causing contamination of water quality and cause pollution. The aim of the study was to present the current situation of healthcare waste management in drugstores and handling pharmacies in the city in study, relating to the current legislation. Data were collected through interviews and questionnaires. The results show that most companies generate about 15 liters and most of this waste is classified as expired medications. The profiles of the companies studied were observed in relation to procedures adopted as final disposal, segregation, training offered to employees and quantification of accidents involving waste.

Keywords: Generation of waste. Minimization of environmental impacts. Management process.

\footnotetext{
${ }^{1}$ Universidade de Passo Fundo (UPF), Passo Fundo, RS. E-mails: (leidalmoro@yahoo.com.br; adalbertopandolfo@hotmail.com; marcelesalles@yahoo.com.br; alinegomes1977@hotmail.com)

2 Complexo de Ensino Superior Meridional (IMED), Passo Fundo, RS. E-mail: (laerciomac@yahoo.com.br)
} 


\section{INTRODUÇÃO}

Os resíduos gerados por estabelecimentos de saúde são um dos principais problemas ambientais, sendo que a recente situação caracteriza-se pelo baixo índice de reciclagem e pela destinação inadequada de sua maior parcela. A implantação de processos de segregação dos diferentes tipos da produção de resíduos em sua fonte e no momento de sua geração conduz à minimização de resíduos, em especial àqueles que requerem um tratamento prévio à disposição final (SCHNEIDER et al. 2004).

O gerenciamento dos resíduos de serviços de saúde implica em cuidados devido à contaminação biológica, química e radioativa de parte desses resíduos, fazendo-se necessários a segregação e o acondicionamento no momento de sua geração. Os benefícios que trará à saúde pública e ao meio ambiente valerá todo o empenho para a implantação de um Plano de Gerenciamento de Resíduos de Serviços de Saúde (TAKADA, 2003, p 17).

O crescimento da população nos últimos anos no município de Marau, localizado no norte do Estado do Rio Grande do Sul, teve como conseqüência o aumento na geração de resíduos, entre eles os Resíduos de Serviço de Saúde (RSS), os quais remetem grandes riscos, aumentando a preocupação com a preservação do meio ambiente, com a saúde pública e a qualidade de vida da população.

De forma geral, é importante referir que além do meio ambiente, um gerenciamento inadequado dos resíduos gerados, tais como: agulhas contaminadas e como matéria prima vencida no processo de manipulação de medicamentos pode causar sérios danos na saúde da população, e também quando há contato sem utilização de equipamentos de proteção adequados. Nesse contexto existem algumas substâncias denominadas poluentes emergentes que aumentam a preocupação e necessitam de uma maximização de cuidados, principalmente quando se fala em resíduos de antibióticos e hormônios. Estes podem ser gerados tanto em farmácias de manipulação como nas drogarias e devem chamar atenção quanto aos riscos relacionados a saúde dos ecossistemas (AMARANTE, RECH, SIEGLOCH, 2017).

No município foram observados problemas referentes ao manejo dos resíduos de serviços de saúde, sendo esses relacionados principalmente à destinação final, no entanto são ocasionados principalmente pela falta de informação dos envolvidos com essa atividade. 
Esta pesquisa visa contribuir com a adequada destinação final dos Resíduos de Serviços de Saúde (RSS) através da análise do sistema de gerenciamento dos resíduos realizado pelas farmácias de manipulação e drogarias da cidade de Marau, RS, além de caracterizá-los, diagnosticando as práticas de gerenciamento dos RSS nesses estabelecimentos.

\section{MATERIAL E MÉTODOS}

A pesquisa foi desenvolvida no município de Marau localizado na região norte do Rio Grande do Sul e tem uma área de 649,3 km², sua população é de 36.600 habitantes, sendo que destes, 31.800 vivem na área urbana e 4.800 vivem no meio rural (FEE, 2010). Por ser um município pioneiro no desenvolvimento da atividade industrial, recebe um grande fluxo de pessoas que migram de seus locais de origem em busca de oportunidade de trabalho, com o objetivo de melhorar a qualidade de vida. O segmento industrial é responsável por $60 \%$ da economia do município, contribuindo para a geração de emprego, seguido pelo setor de serviço responsável por 24,23\%, o comércio, em terceiro lugar, com 13\% e a agropecuária, 2\% (FEE, 2010).

O município possui um total de 22 empresas do ramo farmacêutico: divididas em manipulação e drogarias. As informações foram coletadas em todas as drogarias e farmácias de manipulação localizadas no município, sob a forma de entrevistas e preenchimento de um questionário elaborado para tal pesquisa.

A seguir encontra-se a descrição dos procedimentos adotados para o desenvolvimento da pesquisa.

\subsection{Etapa 1: Diagnóstico do sistema de gerenciamento dos RSS}

Nessa fase foi realizada a análise do sistema por meio das informações coletadas para diagnosticar e detalhar alguns pontos relevantes do gerenciamento dos RSS das drogarias e farmácias de manipulação, entre eles, o uso de paramentação e registros de acidentes ocorridos com os RSS. Com as informações obtidas, essa análise permitiu tecer um perfil das empresas. 


\subsection{Etapa 2: Caracterização do sistema de Gerenciamento de Resíduos de Serviço de Saúde nas farmácias de manipulação e drogarias.}

\section{Fase 2.1: Identificação da quantidade e composição dos RSS gerados}

O universo pesquisado no presente trabalho foi obtido junto a Prefeitura Municipal no setor de cadastro, a informação do nome e endereço de todas as drogarias e farmácias de manipulação da cidade.

Em cada um dos estabelecimentos foram realizadas visitas a fim de aplicar um questionário, o mesmo se caracteriza por conter perguntas abertas e fechadas. As visitas foram agendadas e a aplicação do questionário foi realizada pelos pesquisadores evitando interferências e diminuindo assim possíveis erros.

Nessa fase foi pesquisado o volume gerado mensalmente em litros por cada farmácia, e a composição dos resíduos gerados, (luvas, seringas, máscaras, agulhas, entre outros) através de entrevista e preenchimento do questionário junto com o responsável pelo serviço.

\section{Fase 2.2: Identificação das formas de acondicionamento dos RSS}

Para a identificação das formas de acondicionamento dos resíduos, foram realizadas visitas com a autorização do responsável aos locais utilizados para este fim. Foi analisado se o acondicionamento está de acordo com a norma, apontando: (a) Identificação dos locais para esse fim; (b) Tipos de embalagens utilizadas; (c) Identificação das embalagens.

\section{Fase 2.3: Identificação dos procedimentos de coleta, transporte interno e armazenamento dos RSS}

Nesta fase da pesquisa foram identificados os procedimentos de armazenamento, coleta e transporte interno dos resíduos gerados. Também foi determinada a regularidade, dos horários de coleta destes resíduos. Além de verificar os procedimentos adotados para o transporte interno dos mesmos. Essa identificação foi realizada através das visitas e preenchimento do questionário e após analisada por meio de um gráfico. 


\section{Fase 2.4: Identificação da disposição final e transporte externo dos RSS}

Nesta fase através da entrevista foram identificadas as formas de disposição final dos resíduos e o transporte externo dos mesmos, além do prazo de recolhimento. Essa fase foi realizada por meio de visitas e preenchimento do questionário.

\section{Fase 2.5: Identificação da existência do Plano de Gerenciamento de Resíduos de Serviços de Saúde e licenciamento ambiental nas empresas}

Para a obtenção dessa informação, foram realizadas entrevistas com os responsáveis pelos RSS de cada farmácia, além de uma consulta de dados na Secretaria de Meio Ambiente, sendo verificada a existência da licença ambiental e do PGRSS, se os requisitos descritos são atendidos conforme a legislação pertinente.

\section{RESULTADOS E DISCUSSÃO}

\subsection{Etapa 1: Diagnóstico do sistema de gerenciamento dos RSS}

Com o objetivo de analisar o atual sistema de gerenciamento dos resíduos de serviços de saúde, foi possível classificar as empresas, detalhando o número de funcionários, presença de treinamentos e se a empresa faz a segregação dos resíduos gerados, além do uso da paramentação adequada e se houve acidentes em relação aos resíduos da empresa e o pessoal envolvido. Grande parte das empresas analisadas são drogarias, 86\%, seguido de 14\% classificadas como farmácias de manipulação.

Para contribuir com o perfil das empresas, um dos dados observados foi a data de abertura das mesmas. Grande parte das empresas (36\%) teve o início de suas atividades na década de 1990, e e as demais foram inauguradas após 2010 (27\%).

As empresas visitadas possuem de 3 a 5 funcionários, gerando assim uma incidência de 55\%, são empresas pequenas e com pessoal suficiente para as atividades desenvolvidas.

Como forma de adequar-se a norma em relação ao manuseio dos resíduos evitando acidentes com o pessoal envolvido e contaminação do meio ambiente, algumas das empresas repassam treinamentos. 
Segundo Cussiol (2008), durante o manuseio, há o risco potencial de acidente, principalmente para os profissionais que atuam na coleta, no transporte, no tratamento e na disposição final dos resíduos. Nos treinamentos realizados pelas empresas são aplicadas informações básicas sobre separação dos resíduos, cuidados necessários e as medidas de prevenção, higiene pessoal, dessa forma 36\% das empresas estudadas treinam seus funcionários trimestralmente com relação à segregação, manuseio e acondicionamento dos resíduos e $64 \%$ não treinam seus funcionários. Nesse sentido faz compreender que fica uma lacuna com ausência de informações e que pode vir a ocasionar por falta de conhecimento riscos as pessoas que desempenham funções relacionadas aos resíduos.

Em contraponto muitos docentes, discentes e demais profissionais da área de saúde se preocupam e divulgam a necessidade de serem realizados treinamentos e repassadas todas as informações sobre os resíduos, porém, às vezes, estes realizam o manuseio de forma inadequada (MORESCHI et. al. 2014).

A adequada segregação requer a participação de toda a comunidade. Por isso, os estabelecimentos de saúde devem conscientizar seus colaboradores da sua importância, salientando os aspectos organizacionais como a subdivisão do estabelecimento de saúde, conforme os serviços especializados, classificação dos resíduos sólidos gerados e determinação de responsabilidades aos funcionários (SILVA, 2004).

O modelo de formação de profissionais da área da saúde não prioriza técnicas e práticas preventivas, com isso provoca um certo distanciamento entre a saúde e o ambiente (SOUZA E ANDRADE, 2014).

O objetivo principal da segregação, segundo Ribeiro; Fernandes (2000), não é reduzir a quantidade de resíduos infectantes a qualquer custo, porém criar uma cultura organizacional de segurança e de não desperdício. Quando se faz segregação, é possível uma melhor identificação da geração e uma tomada de decisão para redução e destinação.

Com a preocupação em minimizar a quantidade de resíduos de serviços de saúde, a segregação dos mesmos é realizada em 82\% das empresas, em contrapartida tem-se $18 \%$ que não realizam essa etapa, sendo assim não reduzindo a quantidade, o volume e o risco ocasionado pelos mesmos. Verificou-se que o volume de resíduos contidos nas empresas estava em conformidade com a capacidade de armazenamento, sendo um indicativo da eficiência do sistema de coleta. 
Com o uso da paramentação adequada, há uma diminuição de contaminação ou acidentes envolvendo os resíduos. Os acidentes ocorridos nas empresas relacionando RSS ainda são significativos pela ausência de EPIs necessários ou pelo inadequado uso dos mesmos. No estudo 50\% das empresas não fazem o uso de nenhum EPI para manuseio dos RSS, porém 50\% utilizam luvas seguido de 23 \% com o uso de jalecos. Muitas vezes isso ocorre em função da falta de atenção e controle da própria empresa, que não fornece tais equipamentos necessários.

Mesmo com o uso de EPIs algumas empresas têm registros de acidentes envolvendo funcionário e os resíduos, estes classificados como perfuro cortantes, ou seja, agulha de injetáveis antes ou após aplicação. Esses fatos foram descritos em 2 empresas, ou seja, 9\% dos estabelecimentos estudados. Sendo assim um dos acidentes ocorreu com um funcionário em uma drogaria no período de 3 anos após a abertura da empresa e em outra drogaria após 5 anos de empresa, embora com a aplicação de treinamentos.

De acordo com a NR 32 cabe ao empregador capacitar, inicialmente e de forma continuada, os trabalhadores em todas as etapas do gerenciamento manejo dos resíduos gerados em estabelecimentos de saúde, da mesma forma que dispõe de orientações quanto ao uso de equipamentos de proteção individual (EPIs).

\subsection{Etapa 2: Caracterização do sistema de Gerenciamento de Resíduos de Serviços de Saúde nas farmácias de manipulação e drogarias}

\section{Fase 2.1: Determinação da quantidade e composição dos RSS gerados}

Conforme estudo realizado nas farmácias de manipulação e drogarias da cidade em estudo, no que se refere aos RSS gerados, a quantidade mensalmente gerada em litros está descrita na Tabela 1. Como pode ser observado foi especificado o resultado da análise em volume, pois nas empresas eram apresentados os valores aproximados em litros gerados. 
Tabela 1 - Quantidade total de RSS gerados mensalmente

\begin{tabular}{c|c|c}
\hline $\begin{array}{c}\text { Quantidade de } \\
\text { litros }\end{array}$ & $\begin{array}{c}\text { Quantidade de } \\
\text { empresas }\end{array}$ & Incidência \\
\hline 0 litros & 2 empresas & $9 \%$ \\
Até 15 litros & 17 empresas & $77 \%$ \\
Até 60 litros & 1 empresa & $5 \%$ \\
Até 120 litros & 2 empresas & $9 \%$ \\
\hline
\end{tabular}

Fonte: Autores (2013)

Como pode ser observado na Tabela 1 foram levantadas as incidências relativas à geração dos resíduos produzidos nas 22 unidades prestadoras de serviço, sendo essas farmácias de manipulação e drogarias. Os resultados demonstram que a maioria das empresas geram um volume aproximadamente de 15 litros, representando uma incidência de 77\%. Conforme a RDC n 306, de 7 de dezembro de 2004 da ANVISA, as farmácias de manipulação estão enquadradas como geradores de RSS, indiferente do porte, porém no presente trabalho há duas empresas que declararam não gerar resíduos de serviços de saúde. Em pesquisa divulgada pela ABRELPE (2014), foram coletadas 5.460 toneladas de RSS nos municípios do estado do Rio Grande do Sul, um aumento em torno de 5,6 em relação ao ano de 2013, que apresentou 5.171 toneladas coletados.

De acordo com o estudo realizado, a composição dos RSS se evidencia conforme a Tabela 2 que demonstra a composição dos mesmos.

Tabela 2 - Composição dos RSS gerados nas drogarias e farmácias de manipulação

\begin{tabular}{c|c|c}
\hline Composição & $\begin{array}{c}\text { Quantidade de } \\
\text { empresas }\end{array}$ & Incidência (\%) \\
\hline Medicamento vencido & 15 & 33 \\
Frascos danificados & 8 & 18 \\
Material injetável & 11 & 24 \\
EPi's & 6 & 13 \\
Resíduo de matéria prima & 3 & 7 \\
Nenhum resíduo gerado & 2 & 5 \\
\hline
\end{tabular}

Fonte: Autores (2013)

A Tabela 2 mostra diferentes composições de resíduos gerados, permitindo assim tecer um perfil da empresa. Os resultados demonstram que a maior parte dos resíduos gerados são classificados como medicamentos vencidos, representando 33\%, seguido dos materiais injetáveis totalizando 24\%. Entretanto, duas das empresas diagnosticadas como drogarias informaram que não geram resíduos de serviços de saúde pelo fato de não realizarem a aplicação de injetáveis.

Com relação à geração e classificação dos resíduos, na maioria das empresas os 
resíduos são classificados como medicamentos vencidos, fato justificado nas drogarias, em função do grande volume de medicamentos que não são comercializados dentro do período de validade.

\section{Fase 2.2: Identificação dos procedimentos de transporte interno e armazenamento dos RSS}

O transporte interno é realizado em $100 \%$ das empresas estudadas por funcionários permanentes, fora do horário de manipulação ou maior fluxo de pessoas no caso das drogarias, e estes auxiliares recebem treinamento sobre o manuseio desses resíduos. Como o transporte interno é o translado dos resíduos dos pontos de geração até o local destinado ao armazenamento temporário ou para coleta externa (SILVA, 2004), esse procedimento tem como objetivo garantir a movimentação planejada às áreas de circulação do estabelecimento de saúde, sem oferecer riscos (GUASSÚ, 2007).

$\mathrm{Na}$ pesquisa foram observados que os procedimentos de armazenamento interno são realizados em três etapas, sendo estas: local de origem como em drogarias que aplicam medicamentos injetáveis e depositam os resíduos em caixas apropriadas que ficam no próprio local (sala de injetáveis), ou a empresa não possui local próprio para armazenamento deixando-os no escritório ou almoxarifado ou, então, os resíduos gerados em algumas das empresas são armazenados em sala fechada e identificada adequadamente.

A Resolução da Diretoria Colegiada - RDC no 306 (2004) dispõe que o armazenamento temporário dos recipientes deve ser em local próximo aos pontos de geração, visando agilizar a coleta dentro do estabelecimento, bem como otimizar o translado entre os pontos geradores e a área destinada à apresentação para coleta externa.

Nas empresas visitadas $77 \%$ possui o armazenamento dos resíduos no local de origem, justificado por serem resíduos de medicamentos injetáveis que são depositados no próprio local de aplicação. Em 14\% das empresas que armazenam, conforme a norma, em local fechado e identificado como sala de armazenamento, e 9\% não praticam nenhum tipo de armazenamento temporário. 


\section{Fase 2.3: Identificação das formas de acondicionamento dos RSS}

Quanto às formas de acondicionamento, grande parte das empresas dispõe seu resíduo perfuro cortante em recipiente com paredes rígidas de acordo com a norma. Para evitar possíveis acidentes de funcionários e clientes com os resíduos, $50 \%$ das empresas dispõe os mesmos em recipiente com paredes rígidas, salienta-se também que 41\% não geram resíduos perfuro cortantes, como no caso das farmácias de manipulação e algumas das drogarias e 9\% que não dispõe os resíduos adequadamente.

No que se refere ainda ao acondicionamento, o uso de recipientes com pedal também foi adotado em algumas empresas, $32 \%$ possuem recipiente para armazenamento dos resíduos com pedal facilitando assim a diminuição de contaminação e o manuseio inadequado. Em 68\% das empresas visitadas ainda não dispõe desse sistema. Conforme a norma RDC no 306 da ANVISA (2004), todos os recipientes da empresa devem ser utilizados sem que haja necessidade de abrir com a mão, evitando assim qualquer tipo de contaminação.

De acordo com o estudo realizado, as embalagens utilizadas para 0 acondicionamento dos resíduos estão descritas na Tabela 3, detalhando o número de empresas e a incidência em relação à utilização e a identificação das embalagens para tal etapa, cabe ressaltar que grande parte das empresas utilizam sacos e caixas identificados.

Tabela 3 - Embalagens para o acondicionamento dos RSS nas drogarias e farmácias de manipulação

\begin{tabular}{c|c|c}
\hline Embalagens & $\begin{array}{c}\text { Quantidade de } \\
\text { empresas }\end{array}$ & Incidência(\%) \\
\hline Bombonas & 4 & 18 \\
Caixas descarpak & 8 & 36 \\
Sacos identificados & 8 & 36 \\
Sacos não identificados & 4 & 18 \\
N/A & 2 & 9 \\
\hline
\end{tabular}

Fonte: Autores (2013)

$\mathrm{Na}$ Tabela 3, pode ser analisado que as embalagens utilizadas para 0 acondicionamento dos resíduos são 18\% bombonas, 36\% caixas descarpak, 36\% sacos branco leitoso identificado como resíduo infectante, essa diversidade no uso embalagens esta relacionado com o tipo, quantidade e freqüência dos resíduos gerados, da mesma forma com o tipo de atividade exercida pelo estabelecimento, dessa forma os resíduos dispostos em caixas descarpack são os considerados perfuro - cortantes, que são 
gerados por 24\% das empresas. Nesse sentido pode-se relacionar o tipo de embalagem utilizada com a freqüência de geração desse tipo de resíduo, ou seja, a quantidade gerada. Destacam-se também com $18 \%$ as empresas que utilizam sacos comuns, o que se considera em desacordo com a norma.

\section{Fase 2.4: Identificação da destinação final e transporte externo dos RSS}

Há uma grande preocupação em relação à destinação final dos resíduos, sendo assim grande parte das empresas, totalizando 68\% encaminham o resíduo gerado para tratamento antes da disposição final, contribuindo para a segurança do meio ambiente e da população, como mostra a Tabela 4 e em atendimento a Resolução no 358 (CONAMA, 2005), que dispõe sobre o tratamento e a disposição final dos resíduos de serviços de saúde.

Tabela 4 - Disposição final dos RSS gerados pelas drogarias e farmácias de manipulação

\begin{tabular}{l|c|c}
\hline \multicolumn{1}{c|}{ Disposição final } & $\begin{array}{c}\text { Quantidade de } \\
\text { empresas }\end{array}$ & Incidência (\%) \\
\hline Depósito de resíduo & 4 & 18 \\
urbano & 15 & 68 \\
$\quad$ Terceir. /tratamento & 2 & 9 \\
$\quad$ Não praticado & 1 & 5 \\
\hline
\end{tabular}

Pode ser observado, na Tabela 4, que 18\% das empresas depositam o RSS gerado como resíduo urbano, e o serviço municipal realiza a coleta, porém a maioria tem serviço terceirizado de tratamento. Quanto ao transporte externo e a destinação final dos RSS ficam a cargo de uma empresa terceirizada, mas para os resíduos comuns a coleta é realizada pela prefeitura, que faz a destinação em aterro sanitário licenciado, conforme prevê a Resolução nº 358 (CONAMA, 2005).

Analisou-se, também, se havia envio de algum RSS para a reciclagem. Das 22 empresas analisadas nenhuma encaminha seus RSS para serem reciclados, mas realizam a doação de materiais como papel e papelão, os quais são recolhidos diariamente pelos catadores.

Contrariando o que destacam Schneider et al. (2004), ao considerar o destino de substâncias perigosas, deve-se pensar que estas, antes de serem lançadas no meio ambiente, deverão, primeiramente, passar por uma análise de reutilização, recuperação, 
reciclagem, tratamento ou permanecerem no ambiente de forma segura até sua destinação final.

Em um estudo sobre descarte de medicamentos vencidos realizado na região Serrana de Santa Catarina foi constatado que 40\% das unidades de saúde e 33\% dos hospitais veterinários devolvem para o órgão de comercialização (AMARANTE, RECH, SIEGLOCH, 2017).

No estudo realizado, tomou-se como dado relevante o transporte externo, ou seja, o prazo de recolhimento dos resíduos. Como pode ser observado na Tabela 5, o recolhimento nas empresas é realizado a cada 15 dias, mensal ou a cada 2 meses, porém há a devolução aos fornecedores como é o caso de algumas drogarias, e 18\% que dispõe esses resíduos para coleta pública, entretanto 9\% das drogarias investigadas não efetuam procedimentos de aplicação de injetáveis, dessa forma não geram resíduos. Destaca-se nas empresas o recolhimento mensal, totalizando $36 \%$ e em contra partida a devolução aos fornecedores que totaliza 5\% das empresas estudadas.

Tabela 5 - Transporte externo dos RSS gerados pelas drogarias e farmácias de manipulação

\begin{tabular}{c|c|c}
\hline Transp. Externo & $\begin{array}{c}\text { Quantidade de } \\
\text { empresas }\end{array}$ & Incidência (\%) \\
\hline Nenhum & 2 & 9 \\
Devolução fornecedor & 1 & 5 \\
Quinzenal & 3 & 14 \\
Mensal & 8 & 36 \\
2 meses & 4 & 18 \\
Coleta pública & 4 & 18 \\
\hline
\end{tabular}

Fonte: Autores (2013)

\section{Fase 2.5: Identificação da existência do Plano de Gerenciamento de Resíduos de Serviços de Saúde e Licenciamento Ambiental nas empresas}

O plano de gerenciamento de resíduos de serviços de saúde não está presente em todas as empresas, porém algumas já estão iniciando o processo para implantá-lo, segundo dados observados na pesquisa, 45\% das empresas possuem o PGRSS. Nesse Plano são contemplados itens como: a segregação dos resíduos, o acondicionamento, o armazenamento, o transporte interno e externo, entre outros, conforme o exposto na Resolução nº 358 (CONAMA, 2005).

A partir dos dados obtidos com a pesquisa em Fortaleza-CE, foi possível concluir que a maioria das farmácias entrevistadas possui um plano de gerenciamento de resíduos 
de serviço de saúde, apesar de ainda existirem falhas no manejo dos resíduos. Foi constatado também que falta capacitação e treinamento entre os funcionários dos estabelecimentos (VIDAL, 2012).

Em relação ao licenciamento ambiental, segundo informações obtidas junto à Secretaria do Meio Ambiente de Marau, do total de 20 empresas, 14 são licenciadas, porém algumas estão em fase de vistoria para obtenção da licença.

A inexistência do Plano de Gerenciamento pode ser relacionada à falta de eficiência dos órgãos regulamentadores o que compromete o adequado gerenciamento dos resíduos gerados por estabelecimentos de saúde. (AMARANTE, RECH, SIEGLOCH, 2017)

\section{CONCLUSÕES}

Os resultados obtidos apresentaram a situação das 22 empresas de drogarias e farmácias de manipulação de Marau, RS, por meio de um levantamento de informações acerca do perfil das mesmas e a forma de gerenciamento dos resíduos de serviço de saúde gerados.

Os resultados mostram que há dispariedades nas etapas do gerenciamento em cada empresa estudada, da mesma forma que a quantidade gerada, composição, procedimentos de armazenamento e identificação, o transporte interno, acondicionamento e destinação final. Deve-se ao fato de possuírem atividades e demandas diferentes.

Em relação ao diagnóstico do gerenciamento dos RSS, foi concluído que uma parcela das empresas, repassam para seus colaboradores treinamentos, sendo esses referentes à segregação, manuseio e acondicionamento dos resíduos, a grade maioria das empresas fazem segregação dos resíduos e metade delas não fazem o uso de nenhum EPI para manuseio dos RSS.

Foi realizada também uma avaliação referente à adequação da norma RDC n. 306 da ANVISA nas empresas estudadas. Quanto a adequação da norma destacam-se a segregação dos resíduos realizada pela maioria das empresas estudadas e como falta de adequação referente a norma há a ausência de aplicação de treinamentos aos funcionários, além da grande incidência na ausência do uso de EPIs no manuseio dos resíduos. O PGRSS não está presente em todas as empresas, mas se observou iniciativas de implantação. 
Diante das novas perspectivas de gerenciamento dos RSS, não somente pelas empresas geradoras, cabe à sociedade, em geral, e a cada cidadão a tarefa de apontar as falhas, salientar as atitudes corretas que forem sendo observadas, no sentido de colaborar para que todos possam usufruir de um ambiente saudável e melhor qualidade de vida.

\section{REFERÊNCIAS}

ABRELPE. Panorama dos resíduos sólidos Brasil 2014. Disponível em: www.abrelpe.org.br/Panorama/panorama2014.pdf. Acesso em: 15 maio 2017.

AGÊNCIA NACIONAL DE VIGILÂNCIA SANITÁRIA - ANVISA. Resolução n 306, de 07 de dezembro de 2004. Dispõe sobre o regulamento técnico para o gerenciamento de resíduos de serviços de saúde. Disponível em:

http://www.cfo.org.br/download/pdf/resolucao_rdc_306_2004.pdf. Acesso: em 20 abr. 2012.

Amarante, J.A.S.; Rech, T.D.; Siegloch, A.E. Avaliação do gerenciamento dos resíduos de medicamentos e demais resíduos de serviços de saúde na Região Serrana de Santa Catarina. Revista Engenharia Sanitária Ambiental, v.22, n.2, p. 317-326, mar. Abr. 2017.

BRASIL. Segurança e Saúde no Trabalho em Serviços de Saúde. NR 32. Portaria GM n. ${ }^{\circ}$ 485, de 11 de novembro de 2005. Diário Oficial da União, Brasília, DF, de 16 de Novembro de 2005 b. Disponível em: http://www.guiatrabalhista.com.br/legislacao/nr/nr32.htm. Acesso em: 20 de maio 2017.

CONSELHO NACIONAL DO MEIO AMBIENTE - CONAMA. Resolução n 358, de 29 de abril de 2005. Dispõe sobre o tratamento e a disposição final dos resíduos de serviços de saúde.

Disponível em: www.mma.gov.br/port/conama/res/res05/res35805.pdf. Acesso em: 03 out. 2013.

CUSSIOL, N. A. de M. Manual de gerenciamento de resíduos de serviços de saúde. Belo Horizonte, 2008. Disponível em: <http://www.resol.com.br/cartilha11/feam_manual_grss.pdf>. Acesso em: 28 fev. 2011.

FEE - FUNDAÇÃO DE ECONOMIA E ESTATÍSTICA. Resumo estatístico do município de Marau- RS, dados, 2010, Disponível em: <www.fee.tche.br/setefee/pt/content/resumo/pg-municdetalhe-php¿municipio=Marau> Acesso em 01 de out. de 2012.

GUASSÚ, D. N. Diagnóstico da gestão de resíduos de serviços de saúde gerados no município de Inhapim - MG. Dissertação (Programa de Pós-graduação em Meio Ambiente e Sustentabilidade), Caratinga, Minas Gerais, 2007.

MORESCHI, C.; REMPEL, C.; BACKES, D.S.; CARRENO, I.; SIQUEIRA, D.F.; MARINA, B. A importância dos resíduos de serviços de saúde para docentes, discentes e egressos da área da saúde. Revista Gaúcha de Enfermagem, v. 35, n.2, p. 20-26, 2014.

SCHNEIDER, V. E; Rêgo, R. de C. E.; Caldart, V.; ORLANDIN, S. M. Manual de gerenciamento de resíduos sólidos de serviços de saúde. 2 ed. Caxias do Sul: Educs, 2004. 
SILVA, M. F. I. Resíduos de serviços de saúde: gerenciamento no centro cirúrgico, centro de material e de recuperação anestésica de um hospital no interior paulista. Ribeirão Preto 2004. 107 f. Tese (Doutorado em enfermagem). 2004.

RIBEIRO, F. V. O.; FERNANDES, A. T. Gerenciamento de resíduos de serviços de saúde. infecção hospitalar e suas interfaces na área da saúde. São Paulo, 2000. Disponível em: <www.scielo.br/pdf/esa>. Acesso em: 15 abr. 2011.

SOUZA, C.L. \& ANDRADE, C.S. Saúde, meio ambiente e território: uma discussão necessária na formação em saúde. Ciência e Saúde Coletiva, v. 19, n. 10, p. 4113-4122, 2014.

TAKADA, A. O plano de gerenciamento de resíduos de serviços de saúde e o direito do trabalhado. Brasília, 2003. (Especialização em Direito Sanitário- Escola Nacional de Saúde Pública). 2003.

VIDAL, P. Yasmin. O Gerenciamento dos Resíduos de Serviço de Saúde (RSS) gerados em Farmácias de Fortaleza - CE. In: CONNEPI, 7., 2012. Anais...2012. Disponível em:

$<$ http://propi.ifto.edu.br/ocs/index.php/connepi/vii/paper/viewFile/5237/2395>. Acesso em: 03 out. 2013. 